\title{
A Biphenotypic (Mixed Phenotypic) Acute Leukemia: Report of Two Cases with Immunophenotypic Study
}

\author{
Anupam Sarma ${ }^{1}$, Jagannath Dev Sharma ${ }^{1}$, Chidananda Bhuyan ${ }^{2}$, Munlima Hazarika², \\ Amal Chandra Kataki ${ }^{3}$
}

${ }^{1}$ Department of Pathology, Dr. B. Borooah Cancer Institute, Guwahati, India; ${ }^{2}$ Department of Medical Oncology, Dr. B. Borooah Cancer Institute, Guwahati, India; ${ }^{3}$ Department of Gynecologic Oncology, Dr. B. Borooah Cancer Institute, Guwahati, India. Email: dranupamsarma@gmail.com

Received September $27^{\text {th }}$, 2012; revised October $29^{\text {th }}$, 2012; accepted November $7^{\text {th }}, 2012$

Copyright (c) 2013 Anupam Sarma et al. This is an open access article distributed under the Creative Commons Attribution License, which permits unrestricted use, distribution, and reproduction in any medium, provided the original work is properly cited.

\begin{abstract}
Biphenotypic acute leukemia (BAL) is an uncommon clinical entity. It is a type of acute leukemia with features characteristic of both the myeloid and lymphoid lineages and for this reason is designated as mixed-lineage, hybrid or biphenotypic acute leukemia. As strict diagnostic criteria have only recently been established, the precise incidence among acute leukemia is uncertain, although it is likely to account for approximately less then $5 \%$ of all acute leukemia. BAL is now collectively considered as "mixed phenotype acute leukemia" (MPAL). We hereby report two cases of a rare disease, BAL from our institution in the light of morphology, cytochemistry, flow cytometry and review of literature regarding these cases are described.
\end{abstract}

Keywords: Biphenotypic Acute Leukaemia (BAL); Mixed Phenotype Acute Leukemia (MPAL); Flow Cytometry; Cytochemistry

\section{Introduction}

Diagnosis and classification of acute leukemia depend on morphology, cytochemistry, immunophenotype, cytogenetic and molecular analyses. The examination of the pheripheral blood or bone marrow aspirate and various cytochemical stains, in most of the cases can distinguish lymphoblasts from myeloblasts. The immunophenotyping of the leukemic blasts is essential to distinguish between $\mathrm{B}$ and $\mathrm{T}$ lymphoblastic line and AML type. The immunological markers CD19, CD20, CD22, and CD79a belong to $\mathrm{B}$ cells, while CD2, CD3, CD5, CD7 are found on the surface of the $\mathrm{T}$ cells. Except for M0 and M7 (CD41, CD61), the myeloid markers are CD13, CD33, CD117 and also the myeloperoxidase (MPO). In acute leukemia blast cells frequently express markers of a single cell line (lineage fidelity) and a variable number of cases, an aberrant antigen expression can be found (lineage infidelity).

BAL is a very rare form of hematological malignancy. It is believed to arise from a multipotent progenitor cell and has a poor prognosis. A strict immunophenotypic scoring system has been provided by European Group for Immunological Classification of Leukaemia (EGIL) for its diagnosis. While immunophenotyping is being routinely done to classify acute leukemia, a simple observation of presence of morphologically two distinct variants of blasts in the peripheral blood or bone marrow aspirate may serve as the key to the diagnosis of most cases. A simple myeloperoxidase staining and its positivity in more than $3 \%$ of blasts in the bone marrow may lead to a diagnosis of AML by FAB criteria, so the probability of under diagnosis of BAL cannot be ignored if the facility for immunophenotyping is not available. In this report, we describe two cases of BAL from our institution in the light of morphology, cytochemistry and flow cytometyric immunophenotyping.

\section{Methods}

We report two cases BAL presented to the medical oncology department of Dr. B. Borooah Cancer Institute, Guwahati, a regional institute for treatment and research. After clinical examination blood investigations were carried out. Followed by bone marrow aspiration, bone marrow smears have been prepared and $3 \mathrm{ml}$ aspirated blood taken in EDTA vial. Cytochemical stains are done adjunct to morphological examination. Myeloperoxidase 
(MPO) is located in primary and secondary granules of granulocytes and their precursors. MPO splits hydrogen peroxidase in presence of chromogens like 3,3'diaminobenzidine (DAB). From the bone marrow aspirate in EDTA, flow cytometric immunophenotyping has been done by standard lyse-wash procedure and data was analyzed.

\section{Case History}

\subsection{Case 1}

7 year old male presented with fever, generalized lymphadenopathy (cervical, axillary, and inguinal) for six months. On examination: palor+++, hepatomegaly++. Blood examination: Hb $36 \mathrm{~g} / \mathrm{L}$, WBC $27 \times 10^{9} / \mathrm{L}$, Blasts $95 \%$, Platelet count $100 \times 10^{9} / \mathrm{L}$, LDH $739 \mathrm{U} / \mathrm{L}$, Uric acid $9.6 \mathrm{mg} / \mathrm{dl}$ (Table 1). Peripheral blood showed normocytic normochromic anemia, moderate leukocytosis with preponderance of blasts and moderate thrombocytopenia. The blasts show presence of occasional auer rods in the cytoplasm. Bone marrow aspiration shows hypercellular marrow with two distinct blast populations. One population of blasts is small size with very high N/C ratio, clumped chromatin and inconspicuous nucleoli. The other population of blasts is large in size with dispersed chromatin and prominent nucleoli (Figure 1). Cytochemistry shows $70 \%$ of blasts are MPO positive (Figure 2). From bone marrow aspirate in EDTA, flow cytometric immunophenotyping was carried out by standard lyse-wash procedure and data was analyzed. The abnormal cell cluster was gated (R1) on CD45 which comprises $81.37 \%$ of total leukocyte popultaion. The immunophenotypic score for myloid lineage is 3 (MPO + CD117 $=2+1=3$ ) and the score for B-lymphoid lineage is also 3 (Cyt CD $22+\mathrm{CD} 19=2+1=3$ ). Thus, the features are consistent with BAL according to EGIL criteria (Figure 3). The cytogenetic study was not carried out. The case was planned for blood transfusion and chemotherapy. The patient did not receive treatment and died within one month.

Table 1. Blood examanation of Case 1 and Case 2.

\begin{tabular}{ccc}
\hline Blood examination & Case 1 & Case 2 \\
\hline Haemoglobin & $36 \mathrm{~g} / \mathrm{L}$ & $27 \mathrm{~g} / \mathrm{L}$ \\
WBC count & $27 \times 10^{9} / \mathrm{L}$ & $39.5 \times 10^{9} / \mathrm{L}$ \\
Blasts \% & $95 \%$ & $70 \%$ \\
Platelet count & $100 \times 10^{9} / \mathrm{L}$ & $15 \times 10^{9} / \mathrm{L}$ \\
LDH & $739 \mathrm{U} / \mathrm{L}$ & $1290 \mathrm{U} / \mathrm{L}$ \\
Uric acid & $9.6 \mathrm{mg} / \mathrm{dl}$ & $10.5 \mathrm{mg} / \mathrm{dl}$ \\
\hline
\end{tabular}

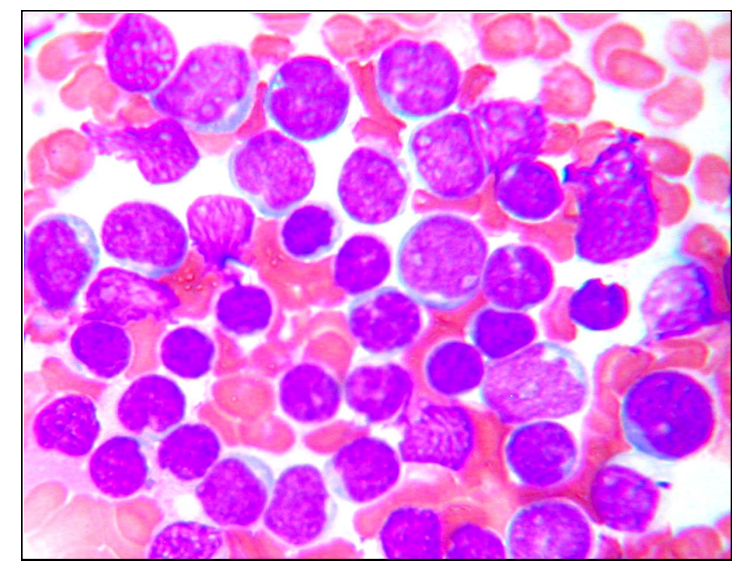

Figure 1. Bone marrow aspiration shows hypercellular marrow with two distinct blast populations. Wright Giemsa stain, $100 \times$.

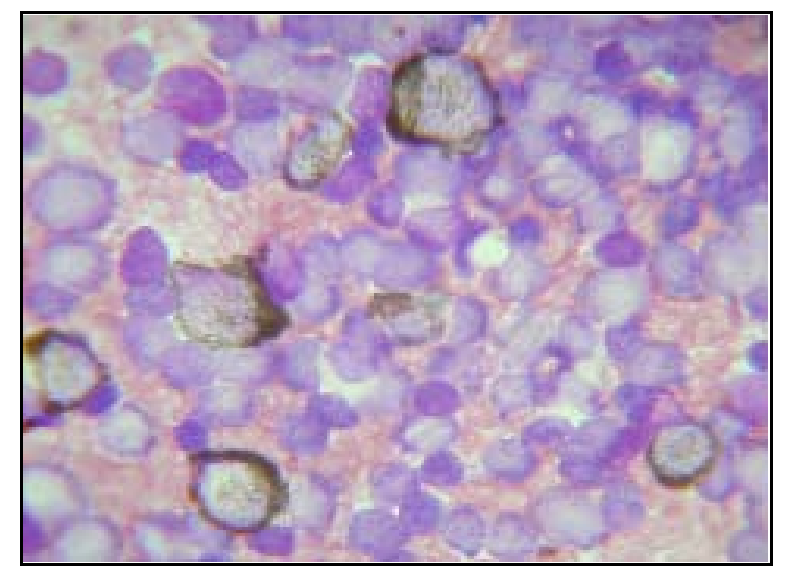

Figure 2. The large blasts are strongly positive for myeloperoxidase (MPO). Substrate used 3,3'diaminobenzidine, Sigma, 100×.

\subsection{Case 2}

55 years old Female presented to the medical oncology clinic at our institution with a history of fever, general weakness for one year. On examination: palor++. Peripheral blood shows moderate leukocytosis with preponderance of blasts, normocytic normochromic anemia with marked thrombocytopenia. Blood examination shows Hb $27 \mathrm{~g} / \mathrm{L}$, WBC $39.5 \times 10^{9} / \mathrm{L}$, Blasts $70 \%$, Platelet count $15 \times 10^{9} / \mathrm{L}$, LDH $1290 \mathrm{U} / \mathrm{L}$, Uric acid 10.5 $\mathrm{mg} / \mathrm{dl}$ (Table 1). Bone marrow aspiration shows intensely hypercellular marrow with preponderance of blasts comprising around $70 \%$ of total nucleated cells with two distinct blast populations. Cytochemistry: $5 \%$ of blasts are MPO positive. From bone marrow aspirate in EDTA immunophenotyping by flow cytometry was carried out in the laboratory. The abnormal cell cluster was gated (R1) on CD45 which comprises $76.96 \%$ of total leukocyte population. The immunophenotypic score for myeloid lineage is $5(\mathrm{MPO}+\mathrm{CD} 117+\mathrm{CD} 13+\mathrm{CD} 33=2+1+1+1=5)$ 

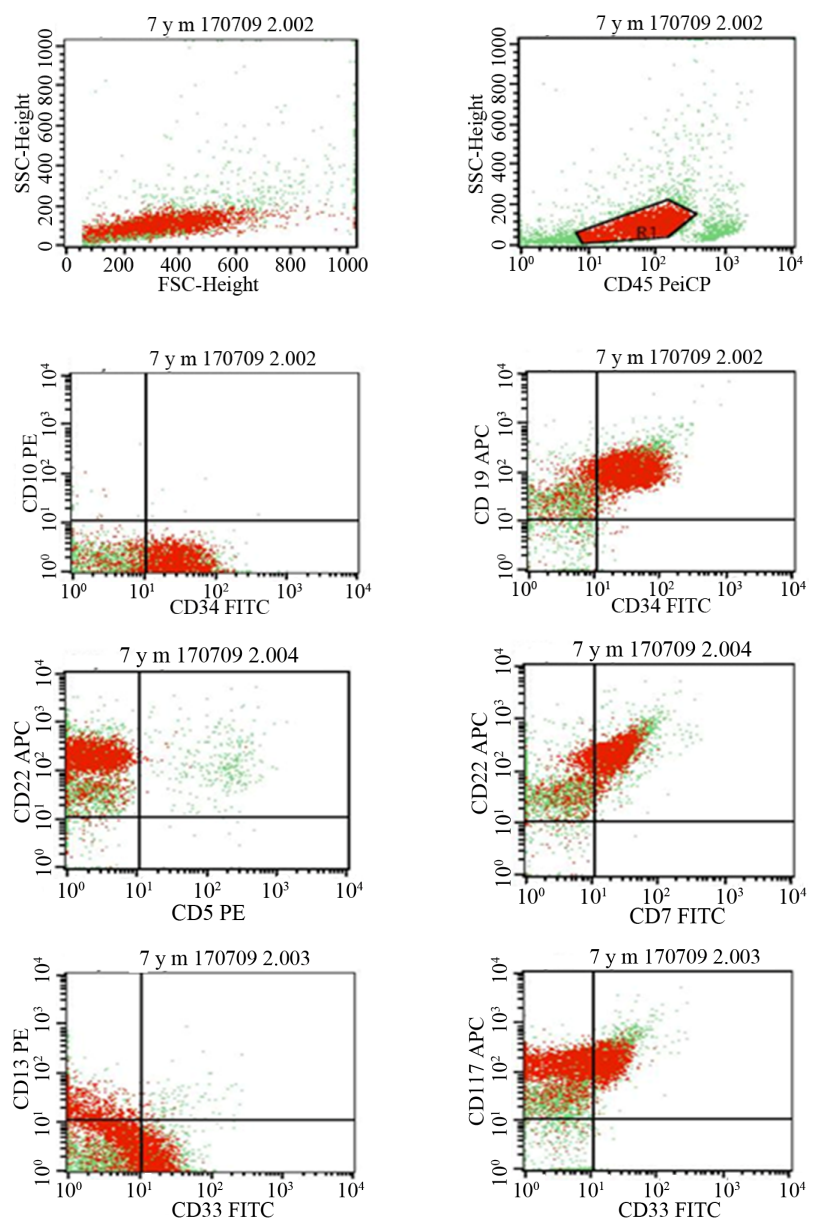

Figure 3. Flow cytometric dot plots: abnormal cell cluster gated (R1) on CD45 which comprises $81.37 \%$ of total leukocyte population demonstrates bright staining for CD34, CD19, CD22, CD117 and weak intensity staining for CD13, CD33, CD7.

and for lymphoid lineage is also 4 (cytCD22 + CD19 + $\mathrm{CD} 10=2+1+1=4)$. The overall impression thus is BAL according to EGIL criteria. The case was taken up for blood transfusion and planed for chemotherapy with ALL regimens but the patient subsequently died.

\section{Discussion}

Acute leukemias can be classified as myeloid, B, or $\mathrm{T}$ lymphoid in origin according to the antigenic profile of the blasts. However, lineage assignment in a minority of cases is not possible because of the evidence of expression of both lymphoid and myeloid lineage-specific antigens in the blast cells. These cases have been described in the literature as biphenotypic acute leukaemia or mixed-lineage acute leukemias and, recently, the designation of mixed phenotype acute leukemia (MPAL) has been proposed by the WHO [1].

As per 2008 World Health Organization (WHO) classification of leukemias, BAL or MPAL is included in the category of acute leukemias of ambiguous lineage. The definition of a BAL is currently based on the scoring system presented by EGIL. According to this scoring system, a diagnosis of acute leukemia of mixed phenotype is established when the score from two separate lineages is each greater than 2 [2].

BAL occurs both in children and adults, but epidemiological data are rare. In a retrospective epidemiological study conducted on 693 adults and children cases with acute leukemia. BAL was detected in only 25 cases (3.6\%) [3]. Amal and et al reported a $4.3 \%$ frequency of BAL in a study in children with acute leukemia. The frequency in children seems to be less than in adult [4]. In our study Retrospective analysis of data of 2 years showed total 72 cases of acute leukemia out of which AML: 24, B-ALL: 39, T-ALL: 07 and BAL: 02 (2.8\%).

Morphologically, BAL is heterogeneous and most patients present either as ALL or as AML with or without myeloid differentiation features. Thus, a diagnosis of BAL is unlikely to be suspected by morphology except for the small subset in which there is evidence of a distinct dualblast population with either lymphoid or myeloid features. Therefore, the diagnosis of BAL always relies on immunophenotyping. In both of our cases bone marrow aspiration shows hypercellular marrow with two distinct blast populations.

According to the EGIL immunological score, several subgroups of BAL have been identified, namely: BAL with B lymphoid and myeloid (B/M) markers co expression, BAL with lymphoid markers $\mathrm{T}$ and $\mathrm{B}(\mathrm{T} / \mathrm{B})$ and BAL with the co expression of $\mathrm{T} / \mathrm{B} /$ myeloid [5]. The most common forms are the BAL B/M, followed by BAL $\mathrm{T} / \mathrm{M}$. BAL T/B is rare and $\mathrm{BAL} \mathrm{T} / \mathrm{B} / \mathrm{M}$ is exceptional [6]. Both of our cases belong to $\mathrm{B}$ lymphoid and myeloid (B/M) category.

The cytogenetic analysis is essential for the prognosis and for the therapeutic attitude in all leukemia cases. The most common cytogenetic anomalies in BAL is $t(9,22)$ (q34; q11) or bcr/abl rearrangements and the rearrangements of MLL (11q23) band, frequently observed in infants with ALL [7].

Extensive data on response to therapy and clinical outcome of BAL is not available; however, cases reported in the literature show a poor outcome in both children and adults. Out of 12 biphenotypic acute leukemia patients at first diagnosis treated with ALL or ALL-based induction regimens, 75\% (9/12) achieved complete remission (CR). Out of 7 patients treated with AML regimens only 2 achieved CR (28.6\%). Of note, 3 of the 5 patients $(60 \%$, $3 / 5$ ) who failed to respond or had only a partial remission (PR) to AML therapy achieved a CR after switching to ALL-based induction therapy. With a median follow-up of 40 months, the median disease-free survival and overall survival were 12 and 16 months. The 3-year dis- 
ease-free survival and overall survival estimates were $28.3 \%$ and $32.4 \%$, respectively [8].

Published studies have documented the poor outcome of BAL in terms of achieving CR and survival and suggested that these are related to association with unfavorable markers such as p-glycoprotein over-expression and unfavorable karyotype. However, these results need to be taken with caution because of the patient's heterogeneity and the small numbers included [9]. In an extensive study of 100 cases of MPAL, it was suggested that ALLdirected treatment seems more effective with a higher response rate and better outcome compared with an AML or to AML combined ALL schedule. In the prognostic risk assessment two other variables might be considered as strong predictors for outcome, age and the presence of $\mathrm{Ph}$ chromosome [10]. Simple immunophenotypic criteria are useful for therapy decisions in MPAL [11].

\section{Conclusion}

BAL/MPAL is a very rare form of hematological malignnancy and represents a diagnostic and therapeutic dilemma. Immunophenotypic criteria are helpful for diagnosis and therapy decisions in BAL/MPAL. The major problem is the choice of therapeutic regime. A unitary therapeutic protocol might be developed in future.

\section{Acknowledgements}

We wish to express our sincere gratitude to Department of Biotechnology, Government of India for their immense support to carry out research works at Dr. B. Borooah Cancer Institute, Guwahati.

\section{REFERENCES}

[1] M. Borowitz, M. C. Bene, N. L. Harris, A. Porwit and E. Matutes, “Acute Leukemias of Ambiguous Lineage," In: S. H. Swerdlow, E. Campo, N. L. Harris, et al., Eds., World Health Organization Classification of Tumours: Pathology and Genetics of Tumours of Haematopoietic and Lymphoid Tissues, IARC Press, Lyon, 2008, pp. 150155.
[2] European Group for the Immunological Classification of Leukaemias, "The Value of c-kit in the Diagnosis of Biphenotypic Acute Leukemia,” Leukemia, Vol. 12, No. 12, 1998, p. 2038. doi:10.1038/sj.leu.2401214

[3] S. Killick, E. Matutes, R. Powles, et al., "Outcome of Biphenotypic Acute Leukemia,” Haematologica, Vol. 84, No. 8, 1999, pp. 699-706.

[4] A. S. Al-Seraihy, M. Tarek, et al., "Clinical Characteristics and Outcome of Children with Biphenotypic Acute Leukemia,” Haematologica, Vol. 94, No. 12, 2009, pp. 1682-1690.

[5] C. A. Hanson, M. Abaza, S. Sheldon, et al., "Acute Biphenotypic Leukemia: Immunophenotypic and Cytogenetic an Analysis," British Journal of Haematology, Vol. 84, No. 1, 1993, pp. 49-60. doi:10.1111/j.1365-2141.1993.tb03024.x

[6] X. Troussard and N. Maarouf, "Leucemies Biphenotypiques: Mythe, Realite, Perspectives,” Spectra Biologie, Vol. 152, 2006, pp. 34-38.

[7] M. C. Bene, "Biphenotypic, Bilineal, Ambigous or Mixed Lineage: Strange Leukemia,” Haematologica, Vol. 94, No. 7, 2009, pp. 891-893. doi:10.3324/haematol.2009.007799

[8] C. C. Zheng, J. S. Wu, X. Liu, et al., "What Is the Optimal Treatment for Biphenotypic Acute Leukemia?” Haematologica, Vol. 94, No. 12, 2009, pp. 1778-1780. doi:10.3324/haematol.2009.014829

[9] O. Legrand, J. Y. Perrot, G. Simonin, et al., “Adult Biphenotypic Acute Leukaemia: An Entity with Poor Prognosis Which Is Related to Unfavourable Cytogenetics and p-Glycoprotein Over-Expression,” British Journal of Haematology, Vol. 100, No. 1, 1998, pp. 147-155. doi:10.1046/j.1365-2141.1998.00523.x

[10] E. Matutes, W. F. Pickl, et al., "Mixed-Phenotype Acute Leukemia: Clinical and Laboratory Features and Outcome in 100 Patients Defined According to the WHO 2008 Classification,” Blood, Vol. 117, No. 11, 2011, pp. 31633171. doi:10.1182/blood-2010-10-314682

[11] E. Mejstrikova, et al., "Prognosis of Children with Mixed Phenotype Acute Leukemia Treated on the Basis of Consistent Immunophenotypic Criteria,” Haematologica, Vol. 95, No. 6, 2010, pp. 928-935. doi:10.3324/haematol.2009.014506 